\title{
Consumo de Canábis: craving e a relação com ansiedade, stresse e depressão
}

\section{Cannabis use: craving and the relationship with anxiety, stress and depression}

\author{
José Vasconcelos-Raposo ${ }^{1}$ \\ Sérgio Couto ${ }^{2}$ \\ Nilton Formiga ${ }^{3}$ \\ Carla M. Teixeira ${ }^{4}$ \\ ${ }^{1}$ Institute for Systems and Computer Engineering, Technology and Science, Portugal \\ ${ }^{2,4}$ Universidade Trás-os-Montes e Alto Douro, Portugal \\ ${ }^{3}$ Universidade Potiguar, Brasil
}

Resumo. A canábis é a substância ilícita mais consumida em Portugal com um início de consumo muito precoce. O craving é considerado um dos factores fundamentais ligados ao consumo de canábis, associando-se à depressão, a ansiedade e ao stresse. Espera-se que nível de craving e sexo se relacionem com a depressão, ansiedade e stresse numa amostra de consumidores portugueses. A amostra foi constituída por 143 consumidores da zona Norte do país e os dados recolhidos através de questionários online (DASS-21 e o MCQ-SF). Os resultados permitem concluir que a emocionalidade, a intencionalidade e a compulsividade relacionaram-se positivamente com o nível do craving, bem como, com o stresse e a depressão. Quanto ao número de anos de consumo, constatou-se um efeito significativo, de dimensão média, na ansiedade.

Palavras chave. Canábis, craving, ansiedade, stress, depressão.

Abstract. Cannabis is the most used illicit substance in Portugal and is the one, which presents, on average, an earlier onset of consumption. Craving is considered one of the fundamental factors linked to the cannabis use, associating anxiety, stress and depression. The sample consisted of 143 individuals from the northern area of the country and the data was gathered through two online questionnaires (DASS-21 and the MCQ-SF). The obtained results allow us to conclude that emotionality, purposefulness and compulsivity are positively associated with the level of craving and, combined, as well to stress and depression. The number of years of consumption presents a significant effect in anxiety, with a medium effect size.

Keywords. Cannabis, craving, anxiety, stress, depression.

\footnotetext{
${ }^{1}$ José Vasconcelos-Raposo. Institute for Systems and Computer Engineering, Technology and Science, Portugal. Endereço Postal: UTAD - Psicologia. Quinta de Prados. 5001-801. Vila Real. Portugal. E-mail: jvraposo@tad.pt

${ }^{2}$ Sérgio Couto. Universidade Trás-os-Montes e Alto Douro, Portugal. E-mail: sergio.e.mendescouto@gmail.com

${ }^{3}$ Nilton Formiga. Universidade Potiguar, Brasil. E-mail: nsformiga@yahoo.com

${ }^{4}$ Carla M. Teixeira. Universidade Trás-os-Montes e Alto Douro, Portugal. E-mail: cteixeir@utad.pt
}

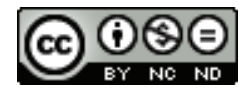

Esta obra está bajo una licencia de Creative Commons Reconocimiento-NoComercial-SinObraDerivada 4.0 Internacional. 


\section{Introducción}

Nas estatísticas da criminalidade ligada à droga, a canábis é a responsável por $80 \%$ das apreensões relatadas e o seu consumo ou posse responsável por $60 \%$ das infracções à legislação em matéria de droga na Europa. No período entre 2006 e 2013, 870 toneladas de canábis foram retiradas de circulação, em resultado das 654000 apreensões que se realizaram. De acordo com o Observatório Europeu da Droga e da Toxicodependência (EMCDDA, 2015), em 2015 foram feitas 732000 que totalizaram 632 toneladas (EMCDDA, 2017). Ainda de acordo com a mesma fonte, a canábis destaca-se, também, pelo seu crescente impacto nos custos do tratamento da toxicodependência, devido ao aumento de pedidos para o tratamento de consumidores desta substância.

A nível europeu, estima-se que na faixa etária entre os 15 e os 65 anos, em 2014, 19.3 milhões de pessoas consumiram canábis (cerca de $5.7 \%$ da população). Se considerarmos a prevalência de consumo de canábis ao longo da vida (pelo menos uma experiência de consumo), o número estimado é de 78.9 milhões de pessoas (cerca de $23.3 \%$ da população).

Em Portugal, no último estudo realizado pelo Serviço de Intervenção nos Comportamentos Aditivos e nas Dependências (SICAD, 2014) os dados referentes à população geral (faixa etária entre os 15 e os 65 anos), a prevalência de consumo de canábis ao longo da vida foi de $9.4 \%$. Cerca de $0.7 \%$ da população geral apresentava sintomas de dependência do consumo de canábis e $0.7 \%$ apresentava um consumo considerado de alto risco (consumo diário ou quase diário de canábis em pelo menos 1 dos últimos 12 meses) (Carapinha, Balsa, Vital, \& Urbano, 2015). No ano de 2013, foram admitidos 1985 novos utentes (usuários) no ambulatório da rede pública, no âmbito do tratamento da toxicodependência, tendo cerca de metade identificado como substância de consumo principal a canábis (SICAD, 2014).

Em estudos realizados na população portuguesa, estima-se que a idade média de início de consumo de canábis seja aos 18 anos, a mais baixa entre as idades médias de início de consumo de substâncias ilícitas. Esta substância é a que apresenta maior consumo ao longo da vida em todas as regiões do país. De acordo com Balsa, Vital e Urbano (2014) há quase uma coincidência entre a experiência de consumo de canábis e a experiência de consumo de qualquer outra substância psicoactiva, ou seja, quase todos os indivíduos que já experimentaram uma substância ilícita já experimentaram canábis.

Atendendo a estes resultados e observações, pode considerar-se o consumo de canábis como um problema de saúde, generalizado a nível europeu. Portugal não é excepção, em particular quando se tem em consideração as prevalências de consumo de alto risco e de dependência. Face a esta realidade o estudo dos factores psicológicos associados ao consumo de canábis assumem particular relevo, para se poder desenvolver políticas de educação que visem a prevenção, assim como para intervenções terapêuticas mais eficazes.

Um dos componentes comportamentais primários da adição a substâncias, nomeadamente à canábis, é o craving, normalmente definido como sendo um desejo ou impulso intenso associado aos efeitos associados ao consumo regular e prolongado no tempo (Filbey, Schacht, Myers, Chavez, \& Hutchison, 2009; Rosenberg, 2009). Outros sugerem que este 
conceito possa ser entendido como sendo uma resposta automática e inconsciente a um estímulo, quer na forma de operacionalização do processamento de informação, quer como a componente de um mecanismo de tomada de decisão.

Para Skinner e Aubin (2010) os triggers (desencadeadores) do craving tanto podem ser biológicos, como psicológicos e motivacionais. Entre várias estratégias para estudar o craving, foram conceptualizdas em quatro dimensões: 1- emocionalidade (uso de canábis por antecipação do alívio da abstinência ou humor negativo), 2- compulsividade (inabilidade para controlar o consumo de canábis), 3- intencionalidade (intenção ou planeamento do consumo de canábis) e 4- expectativa (antecipação de resultados positivos do consumo de canábis) (Heishman \& Singleton, 2006).

Apesar da divergência de definições, existe concordância quanto à importância do craving no consumo de substâncias, em geral, e no consumo de canábis, em particular (Budney, Vandrey, Hughes, Tostenson, \& Bursac, 2008; Dervaux \& Laqueille, 2012; Filbey et al., 2009; Vandrey, Budney, Hughes, \& Liguori, 2008). O craving é considerado um forte preditor do consumo a curto prazo (Serre, Fatseas, Swendsen, \& Auriacombe, 2014) e está, também, associado à manutenção dos consumos, sendo indicado como um sintoma de abstinência (Budney et al., 2008).

A manutenção do consumo de canábis está associado a conjunto de factores quer do foro psicológico como do biológico. De entre os psicológicos mais amplamente citados e tidos como, eventualmente, mais significativos temos a depressão, a ansiedade e o stresse. Dos três, o stresse é referido como sendo um fator de risco predominante para o desenvolvimento de consumos de substâncias (Karoly \& Hutchison, 2012). Isto por que associado ao stresse há a crença que o consumo de canábis é uma estratégia de coping para reduzir o stresse produzido pelas várias circunstâncias que o consumidor frequente enfrenta nas suas vidas (Buckner, Schmidt, Bobadilla, \& Taylor, 2006; Hyman \& Sinha, 2009; Lee, Neighbors, \& Woods, 2007).

A canábis poderá ser consumida por diversos motivos, tendo estes, no entanto, algo em comum: a procura de sensações agradáveis ou de prazer. Contudo, a canábis apresenta vários efeitos adversos tanto agudos como crónicos (Hall \& Degenhart, 2009). A intensidade destes efeitos está associada com a potência da canábis, ou seja, com a concentração do seu componente activo.

O componente activo e constituinte psicoactivo principal da canábis é o $\Delta$ 9-tetrahidrocanabinol (THC) (Dervaux \& Laqueille, 2012) que é responsável pelas sensações de euforia, relaxamento e pela intensificação sensorial procuradas pelos consumidores. No entanto, altas concentrações de THC podem causar crises de pânico, ansiedade, sintomas depressivos e sintomas psicóticos.

O consumo frequente de canábis está, também, associado ao aumento da vulnerabilidade a sintomas de ansiedade e depressão, uma vez que aumenta a reatividade ao stresse e induz sintomas de ansiedade, quer durante o período de intoxicação, quer durante os períodos de abstinência (Stapinski, Montgomery, \& Araya, 2016). A existência de sintomas depressivos, ansiedade e a maior reactividade a situações de stresse leva, por sua vez, a uma manutenção do consumo (Budney et al., 2008). 
O consumo continuado de canábis pode levar à dependência e, consequentemente, ao desenvolvimento de sintomas associados à abstinência da substância. Estes sintomas de abstinência podem manifestar-se num espaço de 24 horas após o consumo, causam grande desconforto e são apontados como um factor importante na recaída de consumidores abstinentes ou em tratamento (Budney et al., 2008). Os consumidores de canábis que procuram tratamento referem diversos sintomas de abstinência, sendo os mais comuns a ansiedade, insónia, irritabilidade e a depressão (Hall \& Degenhart, 2009; Levin et al., 2010; Roura \& Chabrol, 2004).

Contudo, embora os sintomas como a ansiedade e a depressão sejam apontados como efeitos agudos do consumo ou de abstinência, há evidências que sugerem o consumo de canábis como um factor de risco para o desenvolvimento de psicopatologias depressivas e transtornos de ansiedade (Cougle, Hakes, Macatee, Chavarria, \& Zvolensky, 2015). Os estudos sugerem que consumos iniciados na adolescência e que se tornam regulares, frequentemente, estão directamente associados ao aumento da incidência de transtornos depressivos e de ansiedade (Hayatbakhsh et al., 2007). Os consumidores regulares, quando comparados com os ocasionais, apresentam sintomas mais severos de ansiedade e depressão (Horwood et al., 2012; Lubman, Cheetham, \& Yücel, 2015).

Todos estes factores estão relacionados, de uma maneira ou de outra, com o consumo de canábis. No entanto, várias questões continuam por responder, nomeadamente: ¿quais as relações entre esses factores? ¿'Terão a depressão, a ansiedade e o stresse alguma relação com o craving? ¿Poderão outros factores, como o número de anos de consumo ter alguma relação com o craving?

A literatura é escassa ou mesmo inexistente no que diz respeito às respostas a estas questões / relações entre estes factores em consumidores de canábis. Se considerarmos o caso de Portugal, em particular, a investigação com consumidores de canábis é extremamente escassa, embora esta seja a substância ilícita mais consumida no país.

O presente estudo tem como objectivo específico, identificar como o nível de craving se relaciona com a depressão, a ansiedade e o stresse, numa amostra de consumidores portugueses. Pretende-se, ainda, quantificar a relação entre o nível do craving nas diferentes dimensões da escala utilizada para medição do craving (emocionalidade, intencionalidade e compulsividade). Por último, através de comparações, procurámos diferenciar por sexo os consumos e como esses podem ser influenciados pelo número de anos de consumo.

\section{Método}

O presente estudo é um estudo de caráter transversal e correlacional, com um desenho quase-experimental. Os dados foram recolhidos num único momento e analisados através do programa Statistical Package for the Social Science (SPSS), versão 20.

\section{Caracterização da amostra}

A amostra inicialmente recolhida foi composta por 144 indivíduos consumidores de canábis. Durante a análise dos dados, foi verificada a existência de respostas extremas num dos participantes. Os consumidores activos de substâncias ilícitas apresentam 
características especiais e esses particularismos psíquicos podem-se traduzir em respostas com valores extremos devido a exagero ou minimização por parte dos participantes, uma vez que estes procuram, de alguma forma, enquadrar-se no que entendem ser num modelo social de aceitação dos seus comportamentos (Harrell, 1997).

Dada a natureza da recolha de dados efectuada (sem contacto directo com os participantes), não existem informações suficientes que permitam compreender e explicar a existência destes resultados extremos. Por esta razão, optámos por remover os dados dos participantes que apresentaram respostas com assimetrias acentuadas nas respostas aos itens dos questionários. A amostra final foi constituída por 143 participantes, 93 do sexo masculino (65\%) e 50 do sexo feminino (35\%), com idades compreendidas entre os 18 e os 41 anos, com uma média de idades de 25.13 anos e desvio-padrão de 4.41. No que concerne à escolaridade, 1 participante $(0.7 \%)$ finalizou o $4^{\circ}$ ano e, 8 dos participantes $(5.6 \%)$ finalizaram o $6^{\circ}$ ano, $22(15.4 \%)$ finalizaram o $9^{\circ}$ ano, $66(46.2 \%)$ finalizaram o $12^{\circ}$ ano e $46(32.2 \%)$ finalizaram uma licenciatura ou grau superior.

Relativamente à profissão, os participantes foram classificados tendo em conta a Classificação Nacional das Profissões, proposta pelo Instituto Nacional de Estatísticas (INE, 2010). Assim, 5 participantes (3.5\%) integram-se nas profissões ligadas às forças armadas, $44(30.8 \%)$ estudantes, $23(16.1 \%)$ trabalhadores não-qualificados, 4 (2.8\%) operadores de instalações e máquinas e trabalhadores de montagem, 14 (9.8\%) operários, artífices e trabalhadores similares, $3(2.1 \%)$ agricultores e trabalhadores qualificados da agricultura e pescas, $23(16.1 \%)$ pessoal dos serviços e vendedores, $3(2.1 \%)$ pessoal administrativo e similares, $16(11.2 \%)$ técnicos e profissionais de nível intermédio e 8 (5.6\%) especialistas das profissões intelectuais e científicas. Dos participantes, 47 (32.9\%) são estudantes, 65 (45.5\%) encontram-se activos profissionalmente, e 31 (21.7\%) encontram-se desempregados. Foram também analisados dados acerca da frequência de consumo, quantidades e hábitos de consumo.

Relativamente aos consumos efectuados, no que diz respeito à frequência, 87 (60.8\%) referem consumir canábis todos os dias, 28 (19.6\%) consomem canábis de 4 a 6 dias por semana e $28(19.6 \%)$ de 1 a 3 dias por semana. Quando questionados sobre a quantidade consumida diariamente nos últimos 7 dias, $14(9.8 \%)$ referem ter consumido 1 a 2 doses, $10(7 \%)$ referem ter consumido 3 a 5 doses, $30(21 \%) 6$ a 9 doses e $89(62.2 \%)$ referem ter consumido 10 ou mais doses. Quanto ao âmbito de consumo, 10 (7\%) referem consumir exclusivamente sozinhos, $98(68.5 \%)$ referem consumir sozinhos ou em contexto social e $35(24.5 \%)$ referem consumir apenas socialmente.

Da amostra final, todos os participantes eram consumidores de canábis com uma frequência de consumo semanal ou inferior e que já mantinham consumos regulares há períodos de tempo que variavam de 1 a 21 anos, com valor médio de 6.99 anos e desviopadrão 4.08. Quanto à idade de início de consumo regular, esta variou entre os 12 e os 33 anos, com valor médio de 18.14 anos e desvio-padrão 3.31. A divisão da amostra por nível de craving resultou de uma triangulação de dados. Para o efeito recorreu-se à técnica de quartis definindo-se três níveis: baixo $(N=36$; Masc. $=17$; Fem. $=19)$, médio $(N=75$; Masc. $=55 ;$ Fem.; 20) e alto $(N=32 ;$ Masc $=21$; Fem. $=11)$. 


\section{Instrumentos}

Foi elaborado um questionário sócio-demográfico, com perguntas destinadas a uma posterior caracterização da amostra e perguntas destinadas a excluir participantes que não preencham os critérios pretendidos. Os instrumentos de avaliação para medir as variáveis em estudo foram: Marijuana Craving Questionnaire short-form (MCQ-SF) e Depression Anxiety Stress Scales (DASS-21).

Marijuana Craving Questionnaire short-form (MCQ-SF). Adaptado da versão brasileira validada por Pedroso et al. (2014). Este questionário é composto por 12 itens, cotados numa escala de Likert que varia de 1 (concordo fortemente), a 7 (discordo fortemente). Na sua versão alargada esta escala avalia quatro dimensões compulsividade, emocionalidade, expectativa e intencionalidade.

Esta versão, tal como a original, apresenta e elevados de confiabilidade (Heishman et al., 2009). No entanto, a versão de língua portuguesa, que foi utilizada neste estudo, foi dividida em 3 factores, emocionalidade, intencionalidade e compulsividade. No estudo de tradução e adaptação para língua portuguesa todos os factores apresentaram uma consistência interna boa, $\operatorname{com} \alpha=0.79$ para a emocionalidade ( 3 itens), $\alpha=0.67$ para a intencionalidade ( 2 itens) e $\alpha=0.79$ para a compulsividade ( 2 itens).

O resultado total da escala, referente ao nível do craving, é obtido pela soma destes itens com os 5 itens restantes que constituem a escala. Esta versão apresenta boas características psicométricas e é considerada adequada para a avaliação do craving por uso de canábis. A sua consistência interna é satisfatória, apresentando um alfa de Cronbach de 0.89 para o resultado total dos 12 itens da escala (Pedroso et al., 2014).

Esta versão foi utilizada na presente investigação, apresentando esta uma boa consistência interna, com um $\alpha=0.82$ (Maroco \& Garcia-Marques, 2006; Pestana \& Gageiro, 2008). Tendo em conta que o teste Marijuana Craving Questionnaire a origem linguística da versão a utilizar, procedemos à adaptação linguística por forma a obter uma melhor compreensão por parte dos participantes.

Depression Anxiety Stress Scales (DASS-21). Foi adaptada e validada para a população portuguesa por Vasconcelos-Raposo, Fernandes e Teixeira (2013). Esta escala é constituída por 21 itens, distribuídos em 3 dimensões (depressão, ansiedade e stresse), de 7 itens cada. A resposta é dada numa escala do tipo Likert, em que o indivíduo avalia em quatro pontos de gravidade ou frequência, a extensão em que experimentou os sintomas descritos, na última semana. Estes variam de 0 (não se aplicou nada a mim) a 3 (aplicou-se a mim a maior parte das vezes).

Os itens desta escala foram seleccionados para que os resultados possam ser convertidos na pontuação da escala completa, multiplicando estes por dois (PaisRibeiro, Honrado, \& Leal, 2004; Vasconcelos-Raposo et al., 2013). Na versão original da escala utilizada os valores de alfa obtidos forma: Cronbach de 0.84 para a escala de depressão, 0.8 para a ansiedade, 0.82 para o stresse, e um alfa de Cronbach de 0.92 para o resultado total (Vasconcelos-Raposo et al., 2013). Na presente investigação, a escala apresentou uma boa consistência interna (Maroco \& Garcia-Marques, 2006; 
Pestana \& Gageiro, 2008), $\operatorname{com} \alpha=0.81$ para a depressão, $\alpha=0.84$ para a ansiedade, $\alpha=0.80$ para o stresse e $\alpha=0.80$ para o total dos itens da escala.

Osquestionários utilizados foram seleccionados graças às suas características psicométricas assim como pela sua dimensão reduzida e facilidade de compreensão diminuindo, assim, o esforço necessário para responder ao questionário (Rolstad \& Rydén, 2011), por forma a evitar respostas ao acaso ou desistências por parte dos participantes.

Procedimentos e critérios de selecção

Numa primeira fase, foram requisitadas, por correio electrónico, as devidas autorizações para utilização das escalas necessárias. O questionário foi redigido em formato online, através de plataforma específica para o efeito e, posteriormente, publicado em redes sociais. Foi constituído por uma página de informações relativas ao consentimento informado, livre e onde se esclarecia que poderiam desistir de dar respostas a qualquer momento. Tinham de responder a um questionário com variáveis que permitiam a caracterização sócio-demográfica, mas em nenhuma era possível identificar o sujeito. Tendo em conta as características do estudo, o questionário foi publicado de forma a permitir apenas uma resposta por pessoa.

Os critérios de exclusão, para participar da amostra experimental, foram os seguintes: já ter tido um diagnóstico de algum tipo de patologia associada a ansiedade ou depressão; encontrar-se a realizar ou ter já realizado tratamento com o objectivo de cessar ou diminuir o consumo de canábis; consumir canábis por um período inferior a um ano; consumir canábis com uma frequência inferior a semanal; consumir outro tipo de substância ilícita com frequência semanal ou superior; possuir idade inferior a 18 anos.

\section{Procedimentos estatísticos}

Foi realizada uma análise descritiva dos dados, de forma a caracterizar a amostra. A análise de outliers foi realizada através do método das distâncias de Mahalanovis, verificando-se a existência de um outlier, tendo-se considerado pertinente a sua remoção. A variável craving, correspondente ao somatório dos itens do teste MCQ-SF, foi dividida em 3 categorias (baixo, médio e elevado), tendo em conta a análise de quartis. Em seguida, procedeu-se à análise da distribuição da amostra, considerando as variáveis em estudo. Foi possível verificar que as variáveis (emocionalidade, intencionalidade, compulsividade, depressão, ansiedade e stresse) apresentaram distribuição normal, dado que os valores de assimetria e curtose se encontram entre -2 e 2 (Field, 2009; Gravetter \& Wallnau, 2014).

Numa primeira instância optou-se pela análise dos dados utilizando a MANOVA, tendo como variáveis independentes o sexo e os níveis de craving e como variáveis dependentes a emocionalidade, a intencionalidade e a compulsividade. Num segundo momento, foi realizada outra MANOVA com as mesmas variáveis independentes, mas tendo como variáveis dependentes a depressão, a ansiedade e o stresse. O teste $\mathrm{M}$ de Box indicou que existe violação da hipótese de homogeneidade das matrizes de variância-covariância.

No entanto, tendo em conta a sensibilidade deste teste e o facto das variáveis dependentes apresentarem o mesmo $N$, considera-se que a robustez dos resultados da MANOVA se encontra garantida (Tabachnick \& Fidell, 2007). O teste de Levene indicou que existe 
violação do pressuposto de homogeneidade de variância nas variáveis emocionalidade (na $1^{\text {a }}$ análise), ansiedade e stresse (na $2^{\mathrm{a}}$ análise). No entanto, dado o $N$ da amostra ( $N=$ 143) foi considerado elevado e o facto das variáveis dependentes apresentarem o mesmo $N$, a violação deste pressuposto não foi considerada de relevância nem põe em causa a robustez dos resultados da MANOVA (cf. Tabachnick \& Fidell, 2007). Foram realizadas análises post-hoc, recorrendo-se ao teste de Scheffe, para comparar as diferenças das variáveis dependentes entre grupos (nível baixo, moderado e elevado de craving).

Após as análises da MANOVA, foi realizada uma MANCOVA, tendo como variáveis independentes o nível do craving e o sexo, como variáveis dependentes a depressão, a ansiedade e o stresse e como covariável o número de anos de consumo dos participantes. Em seguida, foram realizadas duas regressões lineares múltiplas, após verificação do pressuposto de normalidade dos resíduos através da análise do histograma e do P-P plotde distribuição de resíduos (Dancey \& Reidy, 2006).

Este teste apresenta-se como mais robusto e com melhores capacidades explicativas no que diz respeito à relação entre variáveis, em detrimento dos testes de correlação, como o teste de correlação de Pearson ou o teste de correlação de Spearman (Sheskin, 2000). $\mathrm{Na}$ regressão múltipla foi utilizado o método stepwise. Este método é uma modificação do método forward, no qual todos os regressores inseridos no modelo são reavaliados através de um teste F parcial. Desta forma, regressores redundantes são retirados do modelo (Montgomery, Peck, \& Vining, 2012).

A primeira regressão, teve como variável dependente o nível do craving e como variáveis independentes a emocionalidade, a intencionalidade e a compulsividade. Esta regressão foi realizada com o objectivo de correlacionar os factores da escala utilizada (MCQ-SF) com o nível do craving e calcular a percentagem da variância do nível do craving, explicada por estas variáveis. A segunda regressão teve como variável independente o nível do craving e como variáveis dependentes a depressão, a ansiedade e o stresse. Esta regressão foi realizada com o objectivo de correlacionar as variáveis e calcular a percentagem de variância do nível do craving explicada pela depressão, ansiedade, e stresse.

\section{Resultados}

Os resultados da MANOVA por nível de craving e sexo mostraram diferenças estatisticamente significativas nas comparações por nível de craving $\left(F_{(6,270)}=55.500, p<.001 ; \lambda\right.$ de Wilks $=$ $0.200)$, com um efeito elevado $\left(\eta_{\mathrm{p}}^{2}=0.552\right)$. As comparações por sexo evidenciaram não existir diferenças $F_{(3,135)}=0.710, p=.548 ; \lambda$ de Wilks $\left.=0.984\right)$. Os resultados da interação sexo x craving demonstraram não existir diferenças estatisticamente significativas $\left(F_{(6,270)}\right.$ $=2.233, p=.439 ; \lambda$ de Wilks $=0.958)$.

$\mathrm{Na}$ análise univariada os resultados evidenciaram que ao nível de craving existem efeitos estatísticos muito fortes nas dimensões emocionalidade $\left(F_{(2,137)}=101.451, p<.001, \eta_{\mathrm{p}}{ }^{2}\right.$ $=0.619)$; intencionalidade $\left(F_{(2,137)}=28.302, p<.001 ; \eta_{\mathrm{p}}{ }^{2}=0.292\right)$; e compulsividade $\left(F_{(2,137)}=28.487, p<.001 ; \eta_{\mathrm{p}}^{2}=0.294\right)$, e nos três casos constatou-se com um poder observado muito elevado (1.00) (tabela 1). O nível de craving em associação com o sexo, não apresentou efeitos estatisticamente significativos em nenhuma das variáveis dependentes, 
Tabela 1

Teste de efeitos entre sujeitos I

\begin{tabular}{llccc}
\hline Source & Dependent Variables & $F$ & $p$ & $\eta_{\mathrm{p}}{ }^{2}$ \\
\hline Nível de Craving & Emocionalidade & 101.451 & $<.001$ & .619 \\
& Intencionalidade & 28.302 & $<.001$ & .292 \\
& Compulsividade & 28.487 & $<.001$ & .294 \\
& Depressão & 6.160 & .003 & .083 \\
& Ansiedade & 19.850 & $<.001$ & .225 \\
& Stresse & 17.465 & $<.001$ & .203 \\
\hline
\end{tabular}

se bem que na dimensão emocionalidade fosse sugerido um $\eta_{\mathrm{p}}{ }^{2}=.021$ que representa um efeito pequeno, mas rejeitámos a possibilidade de efeito significativo com base nos seguintes critérios $p=.233$ e PO $=0.310$. Com a aceitação da hipótese nula, evitámos cometer o tipo de erro II.

Tendo-se verificado diferenças entre os três níveis de craving, realizámos uma análise post-hoc, recorrendo ao teste de Scheffe. Os resultados da comparação entre participantes com um nível baixo e moderado de craving evidenciaram diferenças estatisticamente significativas ao nível da emocionalidade ( $p<.001$; IC 95\% -8.64 e -5.88), entre o nível baixo e elevado $(p<.001$; IC 95\% - 10.84 e - 7.53) e entre o nível moderado e elevado $(p=$ .005 ; IC 95\% -3.37 e -0.49). As comparações na dimensão intencionalidade evidenciaram diferenças entre nível baixo e moderado de craving ( $\phi=.047$; IC 95\% - 2.67 e -0.01), entre baixo e elevado, $(\phi<.001$; IC 95\% -6.49 e -3.30 e entre moderado e elevado de craving $(p$ $<.001$; IC 95\% -4.93 e -2.16).

Relativamente à compulsividade, verificámos diferenças estatisticamente significativas entre os participantes com nível baixo e elevado de craving $(\phi<.001$; IC 95\% -6.33 e -3.13), e entre moderado e elevado de craving ( $p<.001$; IC 95\% -5.52 e -2.73). As diferenças encontradas entre o nível baixo e moderado não são interpretáveis face aos resultados apresentados ao nível do intervalo de confiança (IC 95\% entre -1.94 e 0.73): sempre que os sinais dos valores extremos sejam opostos, os resultados não têm significado estatístico.

Os dados do nível de craving e do sexo foram analisados, por meio de uma MANOVA, com os valores de depressão, ansiedade e stresse como variáveis dependentes. Os resultados da MANOVA, por níveis de craving, mostraram que existem diferenças estatisticamente significativas $\left(F_{(6,270)}=12.621, p<.001 ; \lambda\right.$ de Wilks $\left.=0.610\right)$, com um efeito forte $\left(\eta_{\mathrm{p}}^{2}=\right.$ $0.219)$. As comparações por sexo não evidenciaram diferenças $\left(F_{(3,135)}=0.774, p=.511 ; \lambda\right.$ $=0.983)$, assim como as comparações com base na interacção entre craving e sexo $\left(F_{(6,270)}\right.$ $=1.480, p=.032 ; \lambda=1.480)$.

A análise univariada por nível de craving evidenciou diferenças na depressão $\left(F_{(2,137)}=\right.$ $6.160, p=.003 ; \eta_{\mathrm{p}}^{2}=0.083$ (moderado); PO 0.885); ansiedade $\left(F_{(2,137)}=19.850, p<.001\right.$; $\eta_{\mathrm{p}}{ }^{2}=0.225$ (forte); PO 1.00); e stresse $\left(F_{(2,137)}=17.465, p<.001 ; \eta_{\mathrm{p}}{ }^{2}=0.203\right.$ (forte); 1.00) (tabela 2). A interacção entre craving e sexo não produziu quaisquer diferenças. 
A análise post-hoc, recorrendo-se ao teste de Scheffe, para o craving, evidenciou diferenças estatisticamente significativas para a depressão entre participantes com um nível moderado e elevado de craving ( $p=.043$; IC 95\% - 722 e - 0.09). Para a dimensão ansiedade existiram diferenças entre os de nível baixo e moderado ( $\not<.001$; IC 95\% - 12.30 e - 5.41), e entre baixo e elevado ( $p=.004$; IC 95\% -9.73 e -1.48). Relativamente ao stresse, verificou-se diferenças entre o nível baixo e o moderado ( $p<.001$; IC 95\% - 10.08 e - 2.63); entre o nível baixo e elevado ( $\phi<.001$; IC 95\% -15.21 e -6.29); e entre moderado e elevado $(p=$ .022 ; IC $95 \%-8.27$ e - 0.52).

Em seguida, os dados do nível de craving e do sexo foram analisados por meio de uma MANCOVA, tendo como variáveis dependentes o stresse, a depressão e a ansiedade e como covariável o número de anos de consumo. Os resultados mostraram que existe uma diferença estatisticamente significativa, nas comparações por craving $\left(F_{(6,268)}=10.213, p<.001 ; \eta_{\mathrm{p}}^{2}\right.$ $=0.186 ; \lambda$ de Wilks $=0.662 ;$ PO 1.00). As comparações por o sexo não demonstraram diferenças $\left(F_{(3,134)}=0.688, p=.561 ; \lambda\right.$ de Wilks $\left.=0.985\right)$, assim como não houve qualquer efeito aquando da interação entre craving e sexo $\left(F_{(6,268)}=1.414, p=.209 ; \lambda\right.$ de Wilks $\left.=0.940\right)$. A covariável anos de consumo produziu diferenças com efeitos estatísticos moderados $\left(F_{(3,134)}=4.318, p=.006 ; \eta_{\mathrm{p}}^{2}=0.088 ; \lambda\right.$ de Wilks $\left.=0.912 ; \mathrm{PO}=0.859\right)$.

Relativamente ao teste de efeitos entre sujeitos, a análise mostrou que o nível de craving tem efeitos estatisticamente significativos nas variáveis estudadas. O sexo não apresentou efeitos significativos em nenhuma das variáveis dependentes. A análise univariada evidenciou um efeito pequeno na depressão $\left(F_{(2,136)}=4.984, p=.008 ; \eta_{\mathrm{p}}{ }^{2}\right.$ $=0.068)$; moderado na ansiedade $\left(F_{(2,136)}=15.415, p<.001 ; \eta_{\mathrm{p}}{ }^{2}=0.185\right)$ e forte no stresse $\left(F_{(2,136)}=13.105, p<.001 ; \eta_{\mathrm{p}}{ }^{2}=0.162\right)$. O nível de craving em associação com o sexo não apresentou efeitos estatisticamente significativos. O número de anos de consumo tem um efeito estatístico moderado na ansiedade $\left(F_{(1,136)}=8.169, p=.005\right.$; $\eta_{\mathrm{p}}^{2}=0.057$ ) (tabela 2).

Para correlacionar o nível de craving com os valores da emocionalidade, da intencionalidade e da compulsividade, recorreu-se a uma regressão linear múltipla, tendo-se verificado o pressuposto de normalidade dos resíduos da variável dependente (nível de craving) (Dancey \& Reidy, 2006). A regressão múltipla permitiu a criação de um modelo, tendo como preditores a emocionalidade, a intencionalidade e a compulsividade. As variáveis explicativas apresentam

Tabela 2

Teste de efeitos entre sujeitos III

\begin{tabular}{llccc}
\hline Source & Dependent Variables & $F$ & $p$ & $\eta_{p}^{2}$ \\
\hline \multirow{3}{*}{ Nível de Craving } & Depressão & 4.984 & .008 & .068 \\
& Ansiedade & 15.415 & $<.001$ & .185 \\
& Stresse & 13.105 & $<.001$ & .162 \\
& & & & \\
Anos de Consumo & Ansiedade & 8.169 & .005 & .057 \\
\hline
\end{tabular}


uma associação forte com o nível de craving $(R=0.869)$. Considerando o coeficiente de determinação ajustado, é possível verificar que a emocionalidade, a compulsividade e a intencionalidade, juntos, permitem explicar $75.1 \%$ da variância dos níveis de craving.

$\mathrm{Na}$ ANOVA, foram obtidos os seguintes resultados: $F_{(3,139)}=143.468, p<.001$. Estes valores indicam-nos que a probabilidade dos resultados obtidos terem ocorrido por erro amostral é inferior a .001. Foram encontrados valores estatisticamente significativos nos coeficientes não padronizados de regressão; emocionalidade $(B=0.104)$, intencionalidade $(B=0.050)$, e a compulsividade $(B=0.065)$. Tendo em conta o valor de significância do teste $(p<.001)$, é possível afirmar que o coeficiente de regressão é estatisticamente significativo para as três dimensões em estudo.

No que se refere aos valores dos coeficientes padronizados regressão foram obtidos os seguintes, que permitem explicar a maior percentagem de variância do craving: emocionalidade $(\beta=0.669)$, compulsividade $(\beta=0.299)$ e intencionalidade $(\beta=0.227)$. As três variáveis estão positiva e significativamente relacionados com os níveis de craving (tabela 3).

Para correlacionar o nível de craving com os valores da depressão, da ansiedade e do stresse, recorreu-se a uma regressão linear múltipla. A regressão múltipla permitiu a criação de um modelo, tendo como preditores a depressão e o stresse. As variáveis explicativas apresentaram uma associação moderada com o nível de craving $(R=0.501)$. Considerando o coeficiente de determinação ajustado, é possível verificar que a depressão e o stresse, juntos, permitem explicar $24 \%$ da variância dos níveis de craving.

$\mathrm{Na}$ ANOVA, foram obtidos os seguintes resultados: $F_{(2,140)}=23.424, p<.001$. A depressão apresentou um coeficiente de regressão não padronizado de $B=0.022(p=.003)$ e o stresse $B=0.035(p<.001)$, ambos estatisticamente significativos. Os coeficientes de regressão padronizados sugerem que o stresse é a variável que permite explicar a maior percentagem de variância do craving $(\beta=0.426)$, comparativamente à depressão $(\beta=0.221)$ (ver tabela 4$)$.

O método utilizado na regressão linear múltipla excluiu a variável ansiedade, por problemas de colinearidade, uma vez que esta apresentou uma correlação estatisticamente significativa com o stresse. Atendendo aos resultados, verificou-se que a ansiedade não apresentou um coeficiente de regressão estatisticamente significativo $(\phi=.093)$, embora o coeficiente apresente um resultado de Beta $\mathrm{In}=0.131$.

Tabela 3

Coeficientes a da regressão linear múltipla I

\begin{tabular}{llccc}
\hline Model & & $B^{\mathrm{b}}$ & $\beta^{\mathrm{c}}$ & $p$ \\
1 & (Constant) & -.341 & & .005 \\
& Emocionalidade & .104 & .669 & $<.001$ \\
& Compulsividade & .065 & .299 & $<.001$ \\
& Intencionalidade & .050 & .227 & $<.001$ \\
\hline
\end{tabular}

a Variável dependente: Nível de craving

b. Coeficiente não padronizado

c. Coeficiente padronizado 
Tabela 4

Coeficientesa da regressão linear múltipla II

\begin{tabular}{llccc}
\hline Model & & $B^{\mathrm{b}}$ & $\beta^{\mathrm{c}}$ & \multicolumn{1}{c}{$p$} \\
\hline 1 & (Constant) & 1.381 & & $<.001$ \\
& Stresse & .035 & .426 & $<.001$ \\
& Depressão & .022 & .221 & .003 \\
\hline
\end{tabular}

a. Variável dependente: Nível de craving

b. Coeficiente não padronizado

c. Coeficiente padronizado

\section{Discussão}

Com o presente estudo procurámos comparar consumidores de canábis ao nível das variáveis craving e sexo em função da emocionalidade, intencionalidade, compulsividade, depressão, ansiedade e stresse, identificando como estas variáveis contribuem para explicar os níveis de craving vivenciados pelos consumidores que constituem presente amostra.

Os resultados da MANOVA (craving x sexo) evidenciaram diferenças com efeitos muito fortes quando as comparações foram feitas ao nível da variável craving, não se tendo verificado qualquer diferenças nas comparações por sexo, assim como na interacção com um desenho factorial de $3 \times 2$ (craving x sexo). Estes resultados sugerem que são os domínios do foro emocional os que mais explicam o desejo intenso para consumir.

Estes resultados parecem ser reforçados com das comparações realizadas ao nível das restantes três variáveis (depressão, ansiedade e stresse). $\mathrm{Na}$ realidade, os resultados da MANOVA (craving x sexo) ao nível da depressão, ansiedade e stresse evidenciaram existir um efeito muito forte sendo os mais acentuados os que se referem às variáveis ansiedade e stresse e um efeito moderado ao nível da depressão. Quando consideramos a possível interacção das seis variáveis em estudo temos que a emocionalidade e ansiedade são as que apresentam efeitos mais fortes seguidos pelos da intencionalidade e stresse.

A literatura da especialidade apresenta evidências para a relação entre o consumo de canábis e factores como o craving, a depressão, a ansiedade e o stresse (Budney et al., 2008; Levin, et al., 2010). O craving pode apresentar-se como um preditor do consumo de canábis (Serre et al., 2014) ou como um factor que propicia a manutenção do consumo (Budney et al., 2008). A depressão e a ansiedade são mencionadas como efeitos agudos do consumo de canábis, como consequências do consumo continuado, ou como sintomas de abstinência (Levin et al., 2010). O stresse é referido como um factor de risco para o consumo (Karoly \& Hutchison, 2012), estando directamente associado a aumentos na quantidade ou frequência dos consumos (Hyman \& Sinha, 2009).

As comparações que realizámos, por si só, se bem que coerentes entre si, não se apresentaram suficientemente esclarecedoras, daí ser considerado pertinente questionar como e em que dimensão é que cada uma das variáveis estudadas poderia contribuir para o desejo de consumir (craving). Dos resultados apresentados, pode depreender-se que o nível 
de craving apresenta um efeito estatisticamente significativo nos fatores que compõem o MCQ-SF (emocionalidade, intencionalidade e compulsividade).

Os resultados sugerem que quanto mais elevados os valores da emocionalidade, da intencionalidade e da compulsividade, mais elevado será o nível do craving. Estes três factores apresentam associações positivas e estatisticamente significativas com o nível do craving e, combinados, permitem prever $75.1 \%$ da variância do nível do craving, o que indica que a emocionalidade, a intencionalidade e a compulsividade são fortes preditores do nível do craving. Os resultados do nosso estudo corroboram os de estudos anteriores (Heishman \& Singleton, 2006; Pedroso et al., 2014).

Os resultados indicam que quanto mais elevado o nível do craving, maiores os valores do stresse. A associação encontrada entre stresse e o nível do craving, no modelo de regressão, sugerem que o aumento do stresse é acompanhado por um aumento do nível do craving, o que é consistente com o verificado em estudos anteriores (Sinha, 2001; McRae-Clark et al., 2011).

Estes valores relativos ao stresse podem dever-se a um possível consumo de canábis como estratégia de coping, como sugerido por Buckner et al. (2006). Tendo em conta que os participantes mantinham consumos regulares de canábis, deve também ter-se em atenção o facto de que o próprio consumo, segundo Stapinski et al. (2016), está associado a uma maior susceptibilidade ao stresse, o que poderá, parcialmente, explicar os resultados obtidos.

O nível do craving apresenta um efeito significativo na depressão. Os resultados indicam que quanto maior o nível do craving, maiores os valores da depressão. Foi também verificada uma associação positiva entre a depressão e o nível do craving, no modelo de regressão. Este resultado sugere que o aumento de sintomas depressivos poderá estar associado a um aumento do nível do craving, o que corrobora o verificado em estudos anteriores (Elman, Karlsgodt, Gastfriend, Chabris, \& Breiter, 2002; Nakama, et al., 2008; Rao, 2006).

Foi possível verificar que o nível do craving tem um efeito estatisticamente significativo na ansiedade. Os resultados indicam que os participantes com um nível moderado de craving são os que apresentam maior ansiedade, o que contradiz o apresentado em estudos anteriores, que referem que quanto maior o nível de ansiedade, maior o nível do craving (Watson, VanderVeen, Cohen, DeMarree, \& Morrell, 2012) e que o pico deste desejo se faz sentir em situações que desencadeiam a ansiedade social.

Aquando da recolha de dados, nas conversas tidas com os consumidores foi muito frequente constatar que estes relatavam fumar canábis para reduzir a tensão. Esta constatação reforça a interpretação a favor do argumento de que um dos factores motivadores e desencadeadores do uso da canábis é lidar com e reduzir o efeito negativo das situações com que se confrontam (Foster, Ecker, Zvolensky, \& Buckner, 2015).

Uma outra variável tida em consideração foi os anos de consumo. Esta variável apresentou um efeito estatisticamente significativo nos valores da ansiedade, o que sugere que o número de anos de consumo poderá influenciar os valores da ansiedade dos participantes. Estes resultados sugerem que o consumo e, consequentemente, o 
craving resultam de uma aprendizagem em que os consumidores associaram a redução do stresse e ansiedade ao consumo.

Os resultados deste estudo podem contribuir para uma melhor compreensão das variáveis psicológicas associadas ao consumo de canábis e da relação entre elas, contribuindo, assim, para o desenvolvimento de estudos mais aprofundados e específicos, por forma a serem desenvolvidas abordagens e intervenções específicas para esta problemática. Importa não perder de vista que estudos anteriores sugerem que quase um terço dos indivíduos que satisfazem os critérios de diagnóstico para os transtornos de abuso de substâncias também satisfaz os critérios para os transtornos de ansiedade social (Foster et al., 2015).

Há algumas limitações nesta investigação a serem consideradas. A utilização de um teste não-aferido para a população portuguesa, por falta de um teste mais adequado, poderá levantar reservas aos resultados obtidos. Outros aspectos que em estudos futuros deverão ser considerados são: a concentração de THC na canábis consumida; motivos de início de consumo e outros factores psicológicos ou sociais, como a percepção de aprovação parental, e em que medida estes desempenham um papel de mediadores e/ ou preditores dos níveis de craving. A amostra foi recolhida por via electrónica, podendo não ser representativa da população, pelo que as conclusões retiradas dos resultados obtidos focm em particular a amostra estudada, se bem que os vaores dos efeitos nos permitam generalizar à restante população.

\section{Conclusão}

No presente estudo foi possível verificar que o sexo dos participantes não influencia os resultados da emocionalidade, da intencionalidade e da compulsividade. Da mesma forma, não influencia os resultados da depressão, ansiedade, e stresse. A associação entre nível do craving e o sexo também não apresentou um efeito significativo nos resultados das variáveis estudadas podendo, assim, considerar-se que o sexo não é uma variável moderadora ou diferenciadora neste contexto. Relativamente aos anos de consumo, foi encontrado um efeito significativo na ansiedade, o que sugere que o número de anos de consumo poderá influenciar os valores da ansiedade. As associações encontradas evidenciaram que a intensidade do craving foi acompanhada de aumentos na emocionalidade, na compulsividade e na intencionalidade.

Quanto à depressão, ansiedade e stresse, apenas as duas últimas se apresentaram como preditores dos níveis do craving. Ao nível das normas para o tratamento estatístico, a variável ansiedade foi retirada do modelo por problemas de colinearidade. No entanto, os dados sugerem, de forma clara, que há uma associação positiva entre a ansiedade e o nível do craving. Face ao conjunto dos resultados obtidos poderemos concluir que o consumo de canábis é um mecanismo a que os consumidores recorrem para lidar com níveis de depressão, ansiedade e stresse.

\section{Referencias}

Balsa, C., Vital, C., \& Urbano, C. (2014). III Inquérito Nacional ao consumo de substâncias psicoativas na população geral - Portugal 2012. Lisboa: Serviço de Intervenção nos Comportamentos Aditivos e nas Dependências. 
Buckner, J. D., Schmidt, N. B., Bobadilla, L., \& Taylor, J. (2006). Social anxiety and problematic cannabis use: Evaluating the moderating role of stress reactivity and perceived coping. Behaviour Research and Therapy, 44(7), 1007-1015. doi: 10.1016/j. brat.2005.08.002

Budney, A. J., Vandrey, R. G., Hughes, J. R., Tostenson, J. D., \& Bursac, Z. (2008). Comparison of cannabis and tobacco withdrawal: severity and contribution to relapse. Journal of Substance Abuse Treatments, 35(4), 362-368. doi: 10.1016/j. jsat.2008.01.002

Carapinha, L., Balsa, C., Vital, C., \& Urbano, C. (2015). Estimativa do consumo de alto risco de cannabis - Portugal 2012. Lisboa: Serviço de Intervenção nos Comportamentos Aditivos e nas Dependências.

Cougle, J. R., Hakes, J. K., Macatee, R. J., Chavarria, J., \& Zvolensky, M. J. (2015). Quality of life and risk of psychiatric disorders among regular users of alcohol, nicotine, and cannabis: An analysis of the National Epidemiological Survey on Alcohol and Related Conditions (NESARC). Journal of Psychiatric Research, 66-67, 135-141. doi: 10.1016/j.jpsychires.2015.05.004

Dancey, C. P., \& Reidy, J. (2006). Estatística sem Matemática para Psicologia (3a ed.). Porto Alegre: artmed.

Dervaux, A., \& Laqueille, X. (2012). Cannabis; usage et dépendence. La Presse Médicale, 41(12), 1233-1240. doi: 10.1016/j.lpm.2012.07.016

Elman, I., Karlsgodt, K. H., Gastfriend, D. R., Chabris, C. F., \& Breiter, H. C. (2002). Cocaine-primed craving and its relationship to depressive symptomatology in individuals with cocaine dependence. Journal of Psychofarmacology, 16(2), pp. 163167. doi: $10.1177 / 026988110201600207$

Field, A. (2009). Discovering Statistics using SPSS (3rd ed.). London. SAGE.

Filbey, F. M., Schacht, J. P., Myers, U. S., Chavez, R. S., \& Hutchison, K. E. (2009). Marijuana craving in the brain. Proceedings of the National Academy of Science, 106(31), 1301613021. doi: $10.1073 /$ pnas.0903863106

Foster, D. W., Ecker, A. H., Zvolensky, M. J., \& Buckner, J. D. (2015). Social anxiety and cannabis cravings: The influences of parent injunctive norms and tension reduction expectancies. Journal of Social and Clinical Psychology, 34(9), 731-746. doi: 10.1521/jscp.2015.34.9.731

Gravetter, F. J., \& Wallnau, L. B. (2014). Essentials of Statisticsfor the behavioral sciences (9th ed.). Belmont. Wadsworth.

Hall, W., \& Degenhart, L. (2009). Adverse health effects of non-medical cannabis use. Lancet, 374, 1383-1391. doi: 10.1016/S0140-6736(09)61037-0

Harrell, A. V. (1997). The Validity of Self-Reported Drug Use Data: The Accuracy of Responses on Confidential Self-Administered Answered Sheets. NIDA Research Monograph, 167, 37-58. 
Hayatbakhsh, M. R., Najman, J. M., Jamrozik, K., Mamun, A. A., Alati, R., \& Bor, W. (2007). Cannabis and Anxiety and Depression in Young Adults: A Large Prospective Study. Journal of the American Academy of Child \& Adolescent Psychiatry, 46(3). 408417. doi: 10.1097/chi.0b013e31802dc54d

Heishman, S. J., Evans, R. J., Singleton, E. G., Lvin, K. H., Copersino, M. L., \& Gorelick, D. A. (2009). Reliability and Validity of a Short Form of the Marijuana Craving Questionnaire. Drug and Alcohol Dependence, 102(3), 35-40. doi: 10.1016/j.drugalcdep.2008.12.010

Heishman, S. J. \& Singleton, E. G. (2006). Assessment of Cannabis Craving using the Marijuana Craving Questionnaire. Methods in Molecular Medicine 123, 209-216. doi: 10.1385/1-59259-999-0:209

Horwood, L. J., Ferguson, D. M., Coffey, C., Patton, G. C., Tait, R., Smart, D., Letcher, P., Silins, E., \& Hutchinson, D. M. (2012). Cannabis and depression: An integrative data analysis of four Australasian cohorts. Drug and Alcohol Dependence, 126(3), 369-378. doi: 10.1016/j.drugalcdep.2012.06.002

Hyman, S. M., \& Sinha, R. (2009). Stress-related factors in cannabis use and misuse: Implications for prevention and treatment. Journal of Substance Abuse Treatment, 36(4), 400-413. doi: 10.1016/j.jsat.2008.08.005

Instituto Nacional de Estatísitcas. (2010). Classificação Portuguesa das Profissões 2010. Lisboa: Instituto Nacional de Estatística, I. P.

Karoly, H. C., \& Hutchison, K. E. (2012). Does Stress Contribute to the Incubation of Craving? Biological Psychiatry, 71(8), e39. doi: 10.1016/j.biopsych.2011.12.007

Lee, C. M., Neighbors, C., \& Woods, B. A. (2007). Marijuana motives: Young adults' reasons for using marijuana. Addictive Behaviors, 32(7), 1384-1394. doi: 10.1016/j. addbeh.2006.09.010

Levin, K. H., Copersino, M. L., Heishman, S. J., Liu, F., Kelly, D. L., Boggs, D. L., \& Gorelick, D. A. (2010). Cannabis withdrawal symptoms in non-treatmentseeking adult cannabis smokers. Drug and Alcohol Dependence, 111(1), 120-127. doi: 10.1016/j.drugalcdep.2010.04.010

Lubman, D. I., Cheetham, A., \& Yücel, M. (2015). Cannabis and adolescent brain development. Pharmacology \& Therapeutics, 148, 1-16. doi: 10.1016/j. pharmthera.2014.11.009

Maroco, J., \& Garcia-Marques, T. (2006). Qual a fiabilidade do alfa de Cronbach? Questões antigas e soluções modernas? Laboratório de Psicologia, 4(1), 65-90.

McRae-Clark, A. L., Carter, E. R., Price, L. K., Baker, N. L., Thomas, S., Saladin, M. E., Giarla, K., Nicholas, K., \& Brady, K. T. (2011). Stress- and cue-elicited craving and reactivity in marijuana-dependent individuals. Psychopharmacology, 218(1), 4958. doi: 10.1007/s00213-011-2376-3

Montgomery, D. C., Peck, E. A., \& Vining, G. G. (2012). Introduction to Linear Regression Analysis (5 ed.). New Jersey. John Wiley and Sons, Inc. 
Nakama, H., Chang, L., Cloak, C., Jiang, C., Alicata, D., \& Haning, W. (2008). Association between Psychiatric Symptoms and Craving in Methamphetamine Users. American Journal on Addictions, 17(5), 441-446. doi: 10.1080/10550490802268462

Observatório Europeu da Droga e da Toxicodependência. (2015). Relatório Europen sobre Drogas. Luxemburgo: Serviço das Publicações da União Europeia. doi: $10.2810 / 7534$

Observatório Europeu da Droga e da Toxicodependência. (2017). Relatório Europeu sobre Drogas: Tendeências e evoluçôes. Luxemburgo: Serviço das Publicações da União Europeia. doi: 10.2810/4499

Pais-Ribeiro, J. L., Honrado, A., \& Leal, I. (2004). Contribuição para o estudo da adaptação portuguesa das escalas de ansiedade, depressão e stress (EADS) de 21 itens de Lovibond e Lovibond. Psicologia, saúde \& doenças, 5(2), 229-239.

Pedroso, R. S., Castro, M. G., Santos, P. L., Polese, G. P., Balbinot, A. D., Fischer, V. J., Oppermann, C. M. O., \& Araujo, R. B. (2014). Validação psicométrica do Marijuana Craving Questionnaire-Short Form - Versão Brasil. Clinical Biomedical Research, 34(4), 387-396.

Pestana, M. H., \& Gageiro, J. N. (2008). A complementaridade do SPSS (5 ed.). Lisboa: Edições Sílabo.

Rao, U. (2006). Links Between Depression and Substance Abuse in Adolescents: Neurobiological Mechanisms. American Journal of Preventive Medicine, 31(6), 161174. doi: 10.1016/j.amepre.2006.07.002

Rolstad, S. A., \& Rydén, A. (2011). Response Burden and Questionnaire Length: Is Shorter Better? A Review and Meta-analysis. Value in Health, 14(8), 1101-1108. doi: 10.1016/j.jval.2011.06.003

Rosenberg, H. (2009). Clinical and laboratory assessment of the subjective experience of drug craving. Clinical Psychology Review, 29(6), 519-534. doi: 10.1016/j. cpr.2009.06.002

Roura, C., \& Chabrol, H. (2004). Symptômes de tolérance, de sevrage et de dépendance au cannabis chez l'adolescent consommateur. Neuropsychiatrie de l'enfance et de l'adolescence, 52(1), 11-16. doi: 10.1016/j.neurenf.2003.12.006

Serre, F., Fatseas, M., Swendsen, J., \& Auriacombe, M. (2014). Does craving predict substance use for different substance use disorders? An ecological momentary assessment study in patients with alcohol, tobacco, cannabis and heroin dependence. Drug and Alcohol Dependence, 1, e202-e203. doi: 10.1016/j.drugalcdep.2014.02.564

Sheskin, D. J. (2000). Handbook of Parametric and Nonparametric Statistical Procedures (2 ed.). Florida: Chapman \& Hall/ CRC.

Serviço de Intervenção nos Comportamentos Aditivos e nas Dependências. (2014). Relatório Anual 2013 - A situação do País em matéria de drogas e toxicodependências. Lisboa: Serviço de Intervenção nos Comportamentos e nas Dependências. 
Sinha, R. (2001). How does stress increase risk of drug abuse and relapse? Psychopharmacology, 158(4), 343-359. doi: 10.1007/s002130100917

Skinner, M. D., \& Aubin, H. (2010). Craving's place in addiction theory: Contributions of the major models. Neuroscience and Biobehavioral Reviews, 34(4), 606-623. doi: 10.1016/j.neubiorev.2009.11.024

Stapinski, L. A., Montgomery, A. A., \& Araya, R. (2016). Anxiety, depression and risk of cannabis use: Examining the internalising pathway to use among Chilean adolescents. Drug and Alcohol Dependence, 166, 109-115. doi: 10.1016/j. drugalcdep.2016.06.032

Tabachnick, B. G., \& Fidell, L. S. (2007). Using Multivariate Statistics (5th ed.). Boston: Pearson.

Vandrey, R. G., Budney, A. J., Hughes, R. J., \& Liguori, A. (2008). A within-subject comparison of withdrawal symptoms during abstinence from cannabis, tobacco, and both substances. Drug and Alcohol Dependence, 92(1), 48-54. doi: 10.1016/j. drugalcdep.2007.06.010

Vasconcelos-Raposo, J., Fernandes, H. M., \& Teixeira, C. M. (2013). Factor Structure and Reliability of the Depression, Anxiety and Stress Scales in a Large Portuguese Community Sample. Spanish Journal of Psychology, 16(10), 1-10. doi: 10.1017/sjp.2013.15

Watson, N. L., VanderVeen, J. W., Cohen, L. M., DeMarree, K. G., \& Morrell, H. E. (2012). Examining the interrelationships between social anxiety, smoking to cope, and cigarette craving. Addictive Behaviors, 37(8), 986-989. doi: 10.1016/j. addbeh.2012.03.02

Recebido: 24 de março de 2017

Aceitado: 24 de abril de 2018 